\title{
Tolvaptan for the Treatment of Refractory Ascites
}

\author{
Masayuki Kurosaki and Namiki Izumi
}

Key words: tolvaptan, ascites, aquaporin, osmolality, prognosis

\author{
(Intern Med 55: 2909-2910, 2016) \\ (DOI: 10.2169/internalmedicine.55.7556)
}

To the Editor: The findings from a Japanese phase 3 trial revealed that tolvaptan (Otsuka Pharmaceutical, Osaka, Japan), a non-peptide antagonist of the vasopressin type 2 receptor, is effective in the treatment of refractory ascites in cirrhotic patients. Although this agent is now widely used in clinical practice, few reports have so far been published regarding its real-world efficacy. The reported rate of response varies; for example, Akiyama et al. reported a good response (>3-kg reduction in body weight in 4 days) in $46.7 \%$ of subjects (1), Kogiso et al. reported that weight reduction was obtained in $78.6 \%$ of subjects after 6 months of treatment (2), and Ohki et al. reported an effective rate of $63.3 \%$ (>2-kg reduction in body weight or improvement of symptoms) (3). Similarly, Iwamoto et al. evaluated the effect of tolvaptan in 26 patients, and a response was obtained in $80 \%$ ( $>2-\mathrm{kg}$ reduction in body weight after 7 days of treatment) (4). However, these response rates could not be compared between studies, since the definition of a response and the timing of evaluation varied significantly. Standardized criteria defining a response therefore need to be established.

Since not all patients with refractory ascites respond to tolvaptan, identifying a useful predictor of response is important. In the paper by Iwamoto et al., baseline renal function, as reflected by lower urea and creatinine levels, was a significant predictor of response (4). However, the baseline creatinine levels and estimated glomerular filtration rate (eGFR) levels were not associated with response in the study by Ohki et al. (3). Similarly, Akiyama et al. also noted no correlation between the baseline creatinine levels and intermediate-term efficacy. Due to these discrepancies, further larger scale studies may be warranted to validate the association between the renal function and the response to tolvaptan.

However, the decrease in urinary osmolality seems to be a reliable and reproducible on-treatment predictor of response. Iwamoto et al. found that the percentage decrease in urinary osmolality at 4 hours after the administration of tolvaptan was higher in responders $(48.0 \%)$ than in non-responders
(15.4\%) (4). This finding is consistent with those of Ohki et al., who found that a $25 \%$ decrease in urinary osmolality after tolvaptan was a significant predictor of response, with a hazard ratio of 20.7 (3). These observations seem reasonable, since tolvaptan inhibits water reabsorption and promotes the excretion of free water, leading to a decrease in osmolality. Nakanishi et al. studied the dynamic changes in the urinary osmolality and the levels of water channel aquaporin 2 (AQP2) (5). AQP2 is expressed at the apical membrane of renal collecting duct cells, and its expression determines the water permeability and urine concentration. Those authors further found that urinary concentration of AQP2 significantly and rapidly decreased after the administration of tolvaptan, reflecting a selective block of the binding of vasopressin to V2 receptors. The decrease in expression of AQP2 paralleled the decrease in osmolality, but AQP2 expression was more predictive of response than osmolality (5). Urinary AQP2 and osmolality may be the best predictors of response to tolvaptan available at present.

Another important topic for consideration is the effect of tolvaptan on prognosis. A meta-analysis of 5 randomized controlled studies involving 1571 patients revealed that Satavaptan, another V2 receptor antagonist, did not extend survival compared to a placebo (6). Similarly, in the study by Ohki et al., tolvaptan administration significantly reduced the incidence of events related to ascites but did not affect survival (3). In contrast, Iwamoto et al. divided patients with and without response to tolvaptan and found that survival was better in tolvaptan responders than in nonresponders (4). This result seems promising but should be evaluated with caution. Renal function at the baseline was also worse in tolvaptan non-responders, and renal dysfunction is well known to be a significant predictor of poor survival in cirrhotic patients. As those authors mentioned, the prognosis of tolvaptan responders, who had better renal function than non-responders, may have already been good at baseline, irrespective of tolvaptan administration. The impact of tolvaptan on prognosis should be evaluated in a 
matched control study in the future.

Author's disclosure of potential Conflicts of Interest (COI). Masayuki Kurosaki: Honoraria, Otsuka Pharmaceutical. Namiki Izumi: Honoraria, Otsuka Pharmaceutical.

\section{References}

1. Akiyama S, Ikeda K, Sezaki H, et al. Therapeutic effects of shortand intermediate-term tolvaptan administration for refractory ascites in patients with advanced liver cirrhosis. Hepatol Res 45: 1062-1070, 2015.

2. Kogiso T, Tokushige K, Hashimoto E, et al. Safety and efficacy of long-term tolvaptan therapy for decompensated liver cirrhosis. Hepatol Res 46: E194-E200, 2016.

3. Ohki T, Sato K, Yamada T, et al. Efficacy of tolvaptan in patients with refractory ascites in a clinical setting. World J Hepatol 7: 1685-1693, 2015.

4. Iwamoto T, Maeda M, Hisanaga T, et al. Predictors of the effect of tolvaptan on the prognosis of cirrhosis. Internal Medicine 55: 2911-2916, 2016.

5. Nakanishi H, Kurosaki M, Hosokawa T, et al. Urinary excretion of the water channel aquaporin 2 correlated with the pharmacological effect of tolvaptan in cirrhotic patients with ascites. J Gastroenterol 51: 620-627, 2016.

6. Yan L, Xie F, Lu J, et al. The treatment of vasopressin V2receptor antagonists in cirrhosis patients with ascites: a metaanalysis of randomized controlled trials. BMC Gastroenterol 15: 65, 2015.

The Internal Medicine is an Open Access article distributed under the Creative Commons Attribution-NonCommercial-NoDerivatives 4.0 International License. To view the details of this license, please visit (https://creativecommons.org/licenses/ by-nc-nd/4.0/).

(C) 2016 The Japanese Society of Internal Medicine http://www.naika.or.jp/imonline/index.html 